\title{
A Comparison of Mtwo and RaCe Rotary Instruments in the Preparation of Curved Canals
}

\author{
Mimoza Canga ${ }^{1}$, Irene Malagnino ${ }^{2}$, Giulia Malagnino ${ }^{3}$, Vito Malagnino ${ }^{4}$
}

\begin{abstract}
Aim: The aim of this study was to compare the effectiveness of Mtwo and RaCe rotary instruments in cleaning and shaping root canals curvature. Materials and methods: The present study was conducted on 160 simulated canals in resin blocks with an angle curvature of $15^{\circ}-30^{\circ}$. These 160 simulated canals were divided into two groups, where each group consisted of 80 blocks. In the first group, the canals were prepared using Mtwo rotary system (VDW, Munich, Germany). In the second group, the canals were prepared using RaCe instruments (La Chaux-De-Fonds, Switzerland). The data were recorded using SPSS version 23 software (Microsoft, IL, USA).

Results: The results obtained by using the Mtwo rotary instruments showed that these instruments were able to clean and shape in the rightto-left motion curved canals, at different levels, without any deviation and in perfect symmetry, with a $p$ value $=0.000$. The data showed that greater the depth of the root canal, greater the deviations of the RaCe rotary instruments. These deviations occurred in three levels, which are the following: S2 $(p=0.004)$, S3 $(p=0.007)$, and S4 $(p=0.009)$. The Mtwo files can go deeper and create a greater angle in S4 level $\left(21^{\circ}-28^{\circ}\right)$ compared to RaCe instruments with an angle equal to $19^{\circ}-24^{\circ}$.

Conclusion: The present study noted a clinical significant difference between Mtwo rotary instruments and RaCe rotary files used for the canal preparation and indicated that Mtwo instruments are a better choice for the curved canals.

Clinical significance: There are a large number of procedures and instruments used in the preparation of the root canal. Mtwo and RaCe rotary files were the instruments taken under comparison, in order to determine which of them would perform better.

Keywords: Canal curvature, Canal preparation, Mtwo, RaCe, Resin blocks.

The Journal of Contemporary Dental Practice (2020): 10.5005/jp-journals-10024-2751
\end{abstract}

\section{INTRODUCTION}

Root canal preparation using nickel-titanium (NiTi) rotary systems is a great achievement in dentistry, while root canal cleaning is an important step in endodontic therapy. ${ }^{1,2}$ In order to improve the techniques of canal preparation, new endodontic instruments have been created. ${ }^{3,4}$ One of the most successful NiTi rotary systems is Mtwo. It has a cross-section shape in the form of an "italic $S$ " with two cutting blades. The rake angle of Mtwo enhances the cutting efficiency of this instrument. Mtwo tip is noncutting, that is why the variable helical angle reduces the tendency of the instrument to get stucked into the canal. ${ }^{5}$

The basic set of Mtwo rotary files includes four instruments with variable tip sizes ranging from no. 10 to no. 25 , tapers ranging from 0.04 to $0.06-0.07$, and two lengths: 21 and $25 \mathrm{~mm}$. Also file tips range in size from 30,35 , and 40 and tapers of $0.5,0.4$, and 0.7 are available. $^{6}$

Mtwo instruments preserve the original structure of the teeth without doing an early coronal enlargement. Each instrument is used up to the working length without apical pressure. In the moment when a tight contact is sensed by a clinician, the instrument is withdrawn 1-2 mm so that it can be used as a brushing action that will selectively remove the interferences and go toward the apex. ${ }^{7}$

In order to obtain a circumferential cut, the Mtwo instruments are used with a lateral pressing movement. ${ }^{8}$

Plotino et al. ${ }^{9}$ showed that the fatigue of Mtwo instrument was reduced using a lateral brushing. Mtwo instruments have lower risk of instrument fracture and have the ability to clean and shape symmetrically the root canal curvatures, and also due to their S-shaped cross-section, it can perform effectively lateral cutting.
${ }^{1}$ Department of Public Health, Faculty of Public Health, University "Ismail Qemali" Vlora, Albania

2,3Odontoiatric Private Clinic Malagnino, Rome, Italy

${ }^{4}$ Department of Endodontic, The Chief of Endodontic Catheder, University "Gabriele D’Annunzio" Chiety, Italy

Corresponding Author: Mimoza Canga, Department of Public Health, Faculty of Public Health, University "Ismail Qemali" Vlora, Albania, Phone: +355 676502493, e-mail: mimoza-canga@hotmail.com

How to cite this article: Canga M, Malagnino I, Malagnino G, et al. A Comparison of Mtwo and RaCe Rotary Instruments in the Preparation of Curved Canals. J Contemp Dent Pract 2020;21(2):124-128.

Source of support: Nil

Conflict of interest: None

Several studies proved that all Mtwo files should be used for the full length of the root canal. ${ }^{10}$ The specific design and the flexibility of Mtwo instruments make these files effective and safe, so cleaning can be completed in less time..$^{11-13}$

Recently, a new Mtwo instrument design has been introduced (VDW, Munich, Germany). This type of instruments has the same $\mathrm{S}$-shaped cross-sectional design with a noncutting tip. This design is claimed to eliminate threading and binding in continuous rotation and to reduce transportation of debris toward the apex. ${ }^{14}$

The basic series of Mtwo instruments includes eight instruments, with tapers ranging between 0.04 and 0.07 and sizes from ISO 10-40. The manufacturers claim that a crown-down instrumentation sequence is no longer required, since the Mtwo files can be used to the full working length of the root canal as well as to shape the entire length of it, whereas RaCe instruments

(0) The Author(s). 2020 Open Access This article is distributed under the terms of the Creative Commons Attribution 4.0 International License (https://creativecommons. org/licenses/by-nc/4.0/), which permits unrestricted use, distribution, and non-commercial reproduction in any medium, provided you give appropriate credit to the original author(s) and the source, provide a link to the Creative Commons license, and indicate if changes were made. The Creative Commons Public Domain Dedication waiver (http://creativecommons.org/publicdomain/zero/1.0/) applies to the data made available in this article, unless otherwise stated. 
have a triangular alternating cross-sectional cutting, which reduces intraoperative torque values. ${ }^{15}$

The RaCe instruments create dentinal defects that may be related to the cross-sectional design of the files, also with the fact that RaCe instruments have extremely sharp cut ends. According to the manufacturer, the design of the $\mathrm{RaCe}$ instruments reduces the speed and the screw-in effect within the root canal. ${ }^{16}$

The main objective of RaCe instruments is to create larger apical diameters that will help the chemical irritant penetrate better; in this way, the clinician will obtain a greater microbial reduction. ${ }^{17,18}$ Other studies claimed that RaCe system leaves small areas of untouched dentin walls in the middle and cervical thirds. ${ }^{19}$

This purpose of this study was to compare the effectiveness of Mtwo and RaCe rotary instruments in cleaning and shaping root canals curvature, while the objective was to achieve a more biological canal preparation and preserve the anatomic structure of the teeth.

\section{Materials and Methods}

The present study was conducted on 160 simulated canals in resin blocks with an angle curvature of $15^{\circ}-30^{\circ}$. These 160 simulated canals were divided into two groups, where each group consisted of 80 blocks. Each group was divided into two subgroups ( $n=40$ canals each). The remained 80 blocks served as the control group. During the preparation, the blocks were fixed using a container.

The simulated canal subgroups were prepared with Mtwo and RaCe rotary nickel-titanium instruments. All the resin specimens were photographed, before and after instrumentation. The root canals were measured at four different points of reference, starting at $13 \mathrm{~mm}$ from the orifice.

\section{Simulated Canals}

All instruments were used to shape and clean only four simulated canals, using the crown-down technique. Each instrument, before using it was coated into glycerine, which served as a lubricant. Also after the use of each instrument, a copious irrigation with water was done.

Three clinicians conducted the measurements of the canals. After the preparation of the access cavity, the apical patency of the canals was examined using \#10 and \#15 K-files (Mani Co., Tokyo, Japan).

As already mentioned, the sample was divided into two subgroups $(n=40)$. In the first group, the canals were prepared using Mtwo rotary system (VDW, Munich, Germany). The Mtwo files used were as follows: 10/0.04, 15/0.05, 20/0.06, and 25/0.06. These instruments entered in the full length of the canal. Each file was rotated in the canal until it reached the apical point.

In the second group, the canals were prepared using $\mathrm{RaCe}$ instruments (La Chaux-De-Fonds, Switzerland), performing the crown-down technique, using the torque electric control motor (VDWCO, Munich, Germany), with $600 \mathrm{rpm}$ and $2 \mathrm{~N} / \mathrm{cm}$ as follows: $\neq 40 / 0.10, \neq 35 / 0.08, \neq 30 / 0.06, \neq 25 / 0.04$, and $\neq 25 / 0.02$.

According to the instructions of the manufacturer, the RaCe rotary files used were as follows: $40 / 0.10$ at $5 \mathrm{~mm}, 35 / 0.08$ at $7 \mathrm{~mm}$, $30 / 0.06$ at $9 \mathrm{~mm}$, and $25 / 0.04$ at $11 \mathrm{~mm}$, and the instrument $25 / 0.02$ was utilized at $13 \mathrm{~mm}$, which is the full length of the canal.

The examiners took images of the instrumented sample using the optical microscope at a magnification power of 50x, with the help of a Mitutoyo Profile Projector. After that, these images were compared using the autocad program, in order to evaluate the differences before and after the instrumentation, at the four different lengths of the canals.

Based on the results, this study evaluates the shaping ability of these two rotary systems, in relation to specific lengths of the root canals. The results were analyzed using analysis of variance (ANOVA)-test.

The following parameters were taken in consideration observing the four different lengths of the canal.

$\mathrm{A} i$ is the angle of the canal axis, and it was calculated based on the angle formed with the vertical, which passes in the equidistant points of the canal axis, before the preparation.

$\mathrm{RMXi}$ is the distance of the right margin of the canal, from the right side of the resin block.

LMXi is the distance of the left margin of the canal, from the left side of the resin block.

RMXi-LMXi are the distances of the right and the left margins of the canal, evaluated in relation to the right and the left sides of the resin block. This alteration of canal morphology allows us to evaluate the cutting capacity of the instruments.

ai is the angle between the tangent and the canal axis.

After the instrumentation were evaluated the following characteristics:

- Lateral cutting capacity

- Respecting the anatomy of the root canal

Not prepared canals-control group

To standardize the samples, this study took into consideration simulated canal blocks, which were not instrumented $(n=80)$. These blocks were divided into two groups that served as control groups, in order to evaluate the differences between Mtwo and RaCe rotary systems.

The root canal was divided into four heights: $\mathrm{S1}=13 \mathrm{~mm}$, $\mathrm{S} 2=10 \mathrm{~mm}, \mathrm{~S} 3=4 \mathrm{~mm}$, and $\mathrm{S} 4=3 \mathrm{~mm}$ and the canal curvature was evaluated at these four heights in order to determine the greatest change. The data were recorded using SPSS version 23 software (Microsoft, IL, USA). Data analysis was done using ANOVA test.

\section{Results}

Mtwo instruments can facilitate canal preparation. The results obtained by using the Mtwo rotary instruments showed that these instruments were able to clean and shape in the right-to-left motion, at different levels, without any deviation and in perfect symmetry, curved canals, with a $p$ value $=0.000$. Again, based on the results of the present study, Mtwo instruments did not change the original canal curvature and showed good shaping ability in these curved canals. Statistical analysis revealed that Mtwo instruments had the capacity of lateral cutting (Table 1).

The results obtained by using the RaCe rotary instruments showed that these instruments worked asymmetrically from the right to the left motion. The data showed that greater the depth of the root canal, greater the deviations of the RaCe rotary instruments. These deviations occurred in three levels, which are the following: S2 $(p=0.004)$, S3 $(p=0.007)$, and S4 ( $p=0.009)$ (Table 2$)$.

Statistical analysis showed that Mtwo instrument achieved the highest accuracy in $\mathrm{S} 1$ level, because the deviations were equal to zero. While in the other levels: S2, S3, and S4, the deviations were equal to $0.03,0.31$, and 0.43 , respectively. The results of the present study claimed that the Mtwo instruments preserved the original shape of curved canals during preparation (Table 3 ). 
Table 1: $p$ value of different canal lengths (S1-S4) using Mtwo instruments

\begin{tabular}{lc}
\hline $\begin{array}{l}\text { Four different lengths of the } \\
\text { canal treated with Mtwo }\end{array}$ & p value \\
\hline S1-Mtwo-LMXi & 0.000 \\
S1-Mtwo-RMXi & \\
S2-Mtwo-LMXi & 0.000 \\
S2-Mtwo-RMXi & \\
S3-Mtwo-LMXi & 0.000 \\
S3-Mtwo-RMXi & \\
S4-Mtwo-LMXi & 0.000 \\
S4-Mtwo-RMXi & \\
\hline \begin{tabular}{l} 
Table 2: $p$ value of different canal lengths (S1-S4) using RaCe instruments \\
\hline Four different lengths of the \\
canal treated with RaCe
\end{tabular} & \\
\hline S1-RaCe-LMXi & value \\
S1-RaCe-RMXi & 0.000 \\
S2-RaCe-LMXi & \\
S2-RaCe-RMXi & 0.004 \\
S3-RaCe-LMXi & \\
S3-RaCe-RMXi & \\
S4-RaCe-LMXi & \\
S4-RaCe-RMXi & \\
\hline & \\
\hline
\end{tabular}

Table 3: Mean and the standard deviation of canals treated with Mtwo instruments

\begin{tabular}{lcl}
\hline $\begin{array}{l}\text { Canals treated with } \\
\text { Mtwo instruments }\end{array}$ & Mean & Standard deviation \\
\hline S1-Mtwo-LMXi & 0.61 & 0.01 \\
S2-Mtwo-LMXi & 0.50 & 0.01 \\
S3-Mtwo-LMXi & 0.70 & 0.01 \\
S4-Mtwo-LMXi & 1.08 & 0.02 \\
S1-Mtwo-RMXi & 0.61 & 0.01 \\
S2-Mtwo-RMXi & 0.53 & 0.02 \\
S3-Mtwo-RMXi & 1.01 & 0.01 \\
S4-Mtwo-RMXi & 1.51 & 0.02 \\
S1-Mtwo-Ai & 0.02 & 0.01 \\
S2-Mtwo-Ai & 0.08 & 0.004 \\
S3-Mtwo-Ai & 0.67 & 0.007 \\
S4-Mtwo-Ai & 1.25 & 0.01 \\
S1-Mtwo-ai & 0.00 & 0.00 \\
S2-Mtwo-ai & 2.00 & 0.00 \\
S3-Mtwo-ai & 21.00 & 0.00 \\
S4-Mtwo-ai & 28.00 & 0.00 \\
\hline
\end{tabular}

In the root canal treated with $\mathrm{RaCe}$ instruments, the deviations of the S1 level were equal to 0.09 and in S2 level, the deviations were 0.04 , similarly to the deviations of Mtwo instruments (0.03), while in S3 and S4, the deviations of the RaCe instruments were 0.33 and 0.72 (Table 4).

\section{Discussion}

The present study compared the abilities of Mtwo and RaCe rotary instruments in the preparation of curved canals. The process was conducted in laboratory conditions, using simulated resin blocks.
Table 4: Mean and the standard deviation of canals treated with RaCe instruments

\begin{tabular}{lcl}
\hline $\begin{array}{l}\text { Canals treated with RaCe } \\
\text { instruments }\end{array}$ & Mean & Standard deviation \\
\hline S1-RaCe-LMXi & 0.76 & 0.02 \\
S2-RaCe-LMXi & 0.66 & 0.02 \\
S3-RaCe-LMXi & 0.78 & 0.03 \\
S4-RaCe-LMXi & 0.95 & 0.05 \\
S1-RaCe-RMXi & 0.67 & 0.02 \\
S2-RaCe-RMXi & 0.70 & 0.04 \\
S3-RaCe-RMXi & 1.11 & 0.02 \\
S4-RaCe-RMXi & 1.67 & 0.05 \\
S1-RaCe-Ai & 0.10 & 0.01 \\
S2-RaCe-Ai & 0.10 & 0.01 \\
S3-RaCe-Ai & 0.72 & 0.01 \\
S4-RaCe-Ai & 1.29 & 0.01 \\
S1-RaCe-ai & 0.00 & 0.00 \\
S2-RaCe-ai & 2.00 & 0.00 \\
S3-RaCe-ai & 19.00 & 0.00 \\
S4-RaCe-ai & 24.00 & 0.00 \\
\hline
\end{tabular}

Several studies pointed out that the main objective of endodontic is to shape the root canal without any deviation from the original position of the canal. ${ }^{20,21}$

The present research, found out that the Mtwo rotary files used, had the same length and its advantages were the following: the Mtwo files respected the canal anatomy, causing no change to the working length, similar results were obtained by Santoro et al. ${ }^{22}$ Mtwo instruments have a S-shaped cross-sectional design, which reduces the extrusion of debris beyond the apex and has a positive rake angle that can effectively cut the dentin. ${ }^{23}$

This study noticed that Mtwo instruments can equally remove the inner and the outer walls of the canals, thus creates a more anatomic form of the canal. According to the results of this study, the form of the Mtwo instrument pulls the instrument down, whereas the operator should only rotate it. Mtwo instruments work at a rotational speed of $300 \mathrm{rpm}$. Based on the present study observations and analysis, Professor Malagnino noticed that if the speed rotations increase, the instrument will get fractured and the endodontic procedure will fail. The clinicians can enter the canal faster using Mtwo instruments of lower conicity 10/04. Also this research proved that while raising the instrument conicity, the root canals will be cleaned laterally. Mtwo instruments create a conical shape in the apical point, starting from the first millimeter. Based on our findings, Mtwo instruments are able to clean all the diameters and the working length of the canals. Simulated root canals have different angles at different heights and the present study proved that Mtwo instruments respected the axis of canal at each height, from S1 to S4. Mtwo files flexibility helped these instruments follow the angles better.

In order to determine the capacity of Mtwo instruments, this study measured the distance of the right and left margins of the canals.

Veltri et al. ${ }^{24}$ found out that apex anatomy was respected using Mtwo instruments in the apical region. These data were similar to our findings.

In this study, no Mtwo instrument was reported fractured. This finding is in accordance with other studies conducted by Schäfer, 
Vlassis ${ }^{16}$ and Veltri et al. ${ }^{24}$ The results showed that Mtwo instruments caused a greater widening of the root canals. In the S1 level, with a depth of $13 \mathrm{~mm}$ in the root canal, the angle deviation for the Mtwo instruments was $0^{\circ}$, while at S2 level with a depth of $10 \mathrm{~mm}$, the angle deviation was $2^{\circ}$. In the S3 level, Mtwo creates an angle equal to $21^{\circ}$, but these instruments can go deeper and create a greater angle in S4 level $\left(21^{\circ}-28^{\circ}\right)$.

The results showed that $\mathrm{RaCe}$ instruments, in the $\mathrm{S} 1$ level, with a depth of $13 \mathrm{~mm}$ in the root canal, the angle deviation was $0^{\circ}$ while in the S2 level, with a depth of $10 \mathrm{~mm}$, the angle deviation was $2^{\circ}$. In the S3 level, RaCe creates an angle equal to $19^{\circ}$, but these instruments can create a greater angle in the S4 level $\left(19^{\circ}-24^{\circ}\right)$.

The results of this study showed that Mtwo files work with larger angles than RaCe instruments, that is why Mtwo instruments should be taken more in consideration.

$\mathrm{NiTi}$ instrument characteristics, such as elasticity and shape memory, allow Mtwo and RaCe files to preserve the original anatomy of curved canals. ${ }^{25,26}$

Several studies compared the effectiveness of rotary NiTi files and manual instruments in cleaning root canals and they came at the conclusion that NiTi rotary systems are faster than manual files, reduce errors during the preparation of the root canals, and preserve the shape of the root canals. ${ }^{27-29}$

This study proved that Mtwo instruments were highly effective in cleaning and shaping curved canals, and similar results were obtained by, Gu et al., ${ }^{30}$ in their study.

Based on the deviations occurred in the three levels measured, which were S2 $(p=0.004)$, S3 $(p=0.007)$, and S4 $(p=0.009)$, this study can claim that RaCe files were less effective than Mtwo files. Again based on the results, the present study can prove that Mtwo instruments had no deviations at different levels of the canals, and the lateral sides of these canals were in perfect symmetry, with ( $p$ value $=0.000)$

These results reflect that Mtwo rotary files had a better cutting ability, had fewer preparation errors, and had higher flexibility, in comparison to RaCe rotary files.

According to Andrade-Junior et al., ${ }^{31}$ RaCe instruments revealed some canal deviations at all levels, and their findings are similar to the findings of this study.

The RaCe files have a sharp cutting edge with convex triangular cross-section with an asymmetrical longitudinal design. The study observed that RaCe files had different cutting edges on the same file, and this could create stress concentration at specific points which can cause cracks in the instruments. ${ }^{32}$

Garg et al., ${ }^{33}$ in their study conducted on 150 extracted mandibular premolars, showed that cracks were found in $10 \%$ and $16.7 \%$ of the canals prepared with $\mathrm{K} 3$ and RaCe files, respectively.

Merrett et al. ${ }^{34}$ reported fractures of the RaCe files. The present study also reported that four RaCe files were broken, while none of the Mtwo instruments used was fractured.

There are evidences, which prove that NiTi files although have the capacity to preserve the original shape of the canal, they can cause straightening of it if the instrument is left too long within the canal. ${ }^{35}$

Another study conducted by Bürklein et al. ${ }^{36}$ found out that Mtwo instruments maintained the original curvature of the natural teeth, without any deviations.

The present study was done on simulated root canals in resin blocks, and using these simulated canals is an effective method for comparing different root canal instruments. ${ }^{37,38}$
The advantages of using resin blocks include the standardization of the canals' shape and anatomy, the elimination of tooth-related factor, and these resin blocks facilitate the clinicians' work. ${ }^{39}$

Based on the results of this study, Mtwo files performed significantly better in cleaning and shaping the whole canal length.

The process of preparing the root canal curvature becomes more difficult if you get deeper into the root canal. However, this study proved that Mtwo instruments bends better than $\mathrm{RaCe}$ instruments and cleans the canal length better.

This study showed that Mtwo instruments prepared curved canals in a uniform and synchronized way, as it cleans from the left to the right.

\section{Conclusion}

The present study noted a clinical significant difference between Mtwo rotary instruments and RaCe rotary files used for the canal preparation and indicated that Mtwo instruments are a better choice for the curved canals.

\section{Clinical Significance}

There are a large number of procedures and instruments used in the preparation of the root canal. Mtwo and RaCe rotary files were the instruments taken under comparison, in order to determine which of them would perform better.

\section{ACKnOWLedgments}

The author would like to thank my colleagues and in particularly, professor Vito Malagnino, for his support and contribution in conducting this study.

\section{References}

1. Baruah $\mathrm{K}$, Augustine $\mathrm{V}$, Tiwari R, et al. Advances in rotary endodontics - a review. J Adv Med Dent Scie Res 2017;5(7):17-22.

2. Hü Ismann M, Peters OA, Dummer PMH. Mechanical preparation of root canals: shaping goals, techniques and means. Endodontic Topics 2005;10:30-76. DOI: 10.1111/j.1601-1546.2005.00152.x.

3. Kim HC, Kim HJ, Lee CJ, et al. Mechanical response of nickel-titanium instruments with different cross-sectional designs during shaping of simulated curved canals. Int Endod J 2009;42(7):593-602. DOI: 10.1111/j.1365-2591.2009.01553.x.

4. Kuzekanan M. Nickel-titanium rotary instruments: development of the single-file systems. J Int Soc Prev Community Dent 2018;8(5): 386-390. DOI: 10.4103/jispcd.JISPCD_225_18.

5. Malagino VA, Grade NM, Plotino G, et al., The Mtwo NiTi rotary system for root canal preparation. Available at http://www.vdw-dental.com/ pdf/presse/RO0306-59-62-Malagino. PDF 2000.

6. Azar MR, Safi L, Nikaein A. Comparison of the cleaning capacity of Mtwo and Pro taper rotary systems and manual instruments in primary teeth. Dent Res J (Isfahan) 2012;9(2):146-151. DOI: 10.4103/1735-3327.95227.

7. Tang W-R, Smales RJ, Chen H-F, Guo X-Y, et al. Prevention and management of fractured instruments in endodontic treatment World J Surg Proced 2015;5(1):82-98. DOI: 10.5412/wjsp.v5.i1.82.

8. Plotino G, Grande NM, Falanga A, et al. Dentine removal in the coronal portion of root canals following two preparation techniques. Int Endod J 2007;40(11):852-858. DOI: 10.1111/j.1365-2591.2007.01284.x.

9. Plotino G, Grande NM, Sorci E, et al. Influence of a brushing working motion on the fatigue life of NiTi rotary instruments. Int Endod J 2007;40(1):45-51. DOI: 10.1111/j.1365-2591.2006.01179.x.

10. Gavini G, dos Santos M, Caldeira CL, et al. Nickel-titanium instruments in endodontics: a concise review of the state of the art. Braz Oral Res 2018;32(1). DOI: http://dx.doi.org/10.1590/1807-310713. 
11. Schäfer E, Erler M, Dammaschke T. Comparative study on the shaping ability and cleaning efficiency of rotary Mtwo instruments. part a. shaping ability in simulated curved canals. Int Endod J 2006;39(3):196-202. DOI: 10.1111/j.1365-2591.2006.01074.x.

12. Schäfer E, Erler M, Dammaschke T. Comparative study on the shaping ability and cleaning efficiency of rotary Mtwo instruments. part b. cleaning effectiveness and shaping ability in severely curved root canals of extracted teeth. Int Endod J 2006;39(3):203-212. DOI: 10.1111/j.1365-2591.2006.01075.x.

13. Aminsobhani M, Razmi H, Nozari S. Ex Vivo comparison of Mtwo and RaCe rotary file systems in root canal deviation: one file only versus the conventional method. J Dent (Tehran) 2015;12(7):469-477.

14. Kuzekanani M, Walsh LJ, Yousefi MA. Cleaning and shaping curved root canal: MtwoR vs ProtaperR instruments, a lab comparison. Indian J Dent Res 2009;20(3):268-270. DOI: 10.4103/0970-9290.57355.

15. Saberi E, Mohammadi A, Ebrahimipour S, et al. In vitro comparison of NiTi tee, RaCe and Protaper instruments in dentinal crack formation. J dent Mater Tech 2017;6(4):152-158.

16. Schäfer $E$, Vlassis $M$. Comparative investigation of two rotary nickel-titanium instruments: ProTaper versus RaCe. part 2. cleaning effectiveness and shaping ability in severely curved root canals of extracted teeth. Int Endod J 2004;37(4):239-248. DOI: 10.1111/j.01432885.2004.00783.x.

17. García M, Duran-Sindreu F, Mercadé M, et al. A comparison of apical transportation between Profile and RaCe rotary instruments. J Endod 2012;38(7):990-992. DOI: 10.1016/j.joen.2012.03.022.

18. Nabavizadeh $M$, Abbaszadegan $A$, Khojastepour L, et al. A comparison of apical transportation in severely curved canals Induced by Reciproc and Bio RaCe systems. Iran Endod J 2014;9(2):117-122.

19. Busquim S, Cunha RS, Freire L, et al. A micro-computed tomography evaluation of long-oval canal preparation using reciprocating or rotary systems. Int Endod J 2015 Oct;48(10):1001-1006. DOI: 10.1111/ iej.12398.

20. Balani P, Niazi F, Rashid H. A brief review of the methods used to determine the curvature of root canals. J res Dent 2015;3: 57-63.

21. Ponce de Leon Del Bello T, Wang N, Roane JB. Crown-down tip design and shaping. J Endod 2003;29(8):513-518. DOI: 10.1097/00004770200308000-00006.

22. Santoro G, Minozzi F, Migliau G, et al. Comparative evaluation of two NiTi instruments: ProTaper and Mtwo. G It Cons-Supplemento 2006;4:174-175.

23. Guelzow A, Stamm O, Martus P, et al. Comparative study of six rotary nickel-titanium systems and hand instrumentation for root canal preparation. Int Endod J 2005;38(10):743-752. DOI: 10.1111/j.13652591.2005.01010.x.

24. Veltri M, Mollo A, Mantovani L, et al. A comparative study of Endoflarehero Shaper and Mtwo NiTi instruments in the preparation of curved root canals. Int Endod J 2005;38(9):610-616. DOI: 10.1111/j.13652591.2005.00989.x.
25. Silva LA, Leonardo MR, Nelson-Filho $\mathrm{P}$, et al. Comparison of rotary and manual instrumentation techniques on cleaning capacity and instrumentation time in deciduous molars. J Dent Child 2004;71(1):45-47.

26. Kuo $\mathrm{C}$, Wang $\mathrm{Y}$, Chang $\mathrm{H}$, et al. Application of ni-ti rotary files for pulpectomy in primary molars. J Dent Sci 2006;1:10-15.

27. Crespo S, Cortes O, Garcia C, et al. Comparison between rotary and manual instrumentation in primary teeth. J Clin Pediatr Dent 2008;32(4):295-298. DOI: 10.17796/jcpd.32.4.157136355u606576.

28. Soares F, Varella CH, Pileggi R, et al. Impact of er,cr: YSGG laser therapy on the cleanliness of the root canal walls of primary teeth. J Endod 2008;34(4):474-477. DOI: 10.1016/j.joen.2008.02.006.

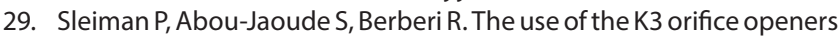
in primary teeth preparation. Oral Health 2007;97:17-18.

30. Gu YX, Zhu YQ, Du R. A comparative study of three different rotary $\mathrm{NiTi}$ systems in the preparation of curved molar canals. Shanghai Kou Qiang Yi Xue 2009;18(2):147-151.

31. Andrade-Junior VC, Dessaune Neto N, Costa-Val Rodrigues R, et al. Transportation assessment in simulated curved canals after preparation with twisted file adaptive and BT-race instruments'. 2017;9(9):e1136-e1140. DOI: 10.4317/jced.54220.

32. Devale RM, Mahesh MC, Bhandary SH. Effect of instrumentation length and instrumentation systems: hand versus rotary files on apical crack formation - an in vitro study. J Clin Diagn Res 2017;11(1):ZC15-ZC18. DOI: 10.7860/JCDR/2017/22059.9159.

33. Garg $S$, Mahajan $P$, Thaman D, et al. Comparison of dentinal damage induced by different nickel-titanium rotary instruments during canal preparation: an in vitro study. J Conserv Dent 2015;18:302-305. DOI: 10.4103/0972-0707.159730.

34. Merrett SJ, Bryant ST, Dummer PM. Comparison of the shaping ability of RaCe and FlexMaster rotary nickel-titanium systems in simulated canals. J Endod 2006;32(10):960-962. DOI: 10.1016/j.joen.2006.03.002.

35. Baumann MA. Nickel-titanium: options and challenges. Dent Clin N Am 2000;48:55-67. DOI: 10.1016/j.cden.2003.11.001.

36. Bürklein S, Hinschitza K, Dammaschke T, et al. Shaping ability and cleaning effectiveness of two single-file systems in severely curved root canals of extracted teeth: reciproc and waveone versus Mtwo and protaper. Int Endod J 2012;45(5):449-461. DOI: 10.1111/j.13652591.2011.01996.x.

37. Baumann MA, Roth A. Effect of experience on quality of canal preparation with rotary nickel-titanium files. Oral Surg Oral Med Oral Pathol Oral Radiol Endod 1999;88(6):714-718. DOI: 10.1016/ S1079-2104(99)70015-6.

38. Schäfer E, Lohmann D. Efficiency of rotary nickel-titanium flexmaster instruments compared with stainless steel hand k-flexofilepart 1. shaping ability in simulated curved canals. Int Endod J 2002;35(6):505-513. DOI: 10.1046/j.1365-2591.2002.00513.x.

39. Goldberg M, Dahan S, Machtou P. Centering ability and influence of experience when using waveone single-file technique in simulated canals. Int J Dent 2012;2012:206321. DOI: 10.1155/2012/206321. 